\title{
Research and Application of Power Supply Enterprise Information System Disaster Recovery Technology
}

\author{
Quan Sun*, Yuan Gao, Guofeng Xing, Bo Sun, Chunyu Ni \\ State Grid Tieling Electric Power Supply Company, Tieling, Liaoning Province, China \\ xxxx@Springer.com
}

\begin{abstract}
With the rapid development of the power supply enterprise, research on information disaster recovery system of power supply enterprise has attracted more and more attention. In order to ensure the safety of the power supply enterprise information system for massive data, an information disaster recovery system of power grid enterprises is proposed in this paper, the key technologies of the disaster recovery are analyzed, then according to the selection criteria of the disaster recovery plan, the disaster recovery system of power supply enterprises is designed. The experimental results show that this system can be used for the power supply enterprise data backup, and achieve the zero data loss, so as to ensure the security of information, which has important practical significance for the construction of disaster recovery system.
\end{abstract}

Keywords: Power supply enterprise; disaster recovery; backup strategy.

\section{Introduction}

In recent years, with the rapid development of the power grid enterprise, enterprise database backup has become one of the most important factors that influence the development of the enterprise. Foreign related researches started earlier, after decades of development, has formed a relatively mature technologies and theories. Although domestic related researches started late, but in recent years, some progress has been made. The disaster recovery backup project of the power grid dispatching automation system was proposed by Xiong Wen in 2005, the scheme improved the operation reliability and stability of the dispatching automation system [1]. The network disaster tolerance control and management system was designed by $\mathrm{Hu}$ Yonghua in 2010, the results shown that the system had better applicability and applicability and provided a theoretical basis for other disaster recovery system research [2]. The grid dispatching standby system and information disaster recovery system were proposed by Wang Muwei in 2011, the system ensured the normal and stable operation of power network dispatching [3]. The enterprise automation system of power network safety operation and disaster recovery and backup system were put forward by Lou Shuqing in 2010, experimental results shown that the system had high adaptability and applicability [4]. The electric power dispatching center of the design of the electric power dispatching center disaster recovery system was proposed by Zhang Liang in 2013, experimental results shown that the system could meet the power grid enterprise information system disaster tolerance requirements and had a good promotion [5]. However, most of the current disaster recovery system doesn't take into account the complexity and particularity of the power supply enterprise information system, and structure is not reasonable and stable enough, and the cost is high. 
In order to ensure the safety of mass data in the information system of the power supply enterprise, this paper designs the power grid enterprise information disaster recovery and backup system. The remainder of this paper is organized as follows. Section 2 describes the relevant theories and techniques. Section 3 gives the study on the power supply enterprise information system disaster recovery technology. Section 4 presents a real experiment to evaluate the method. Conclusion is summarized in Section 5.

\section{State of the Art}

The various aspects of the information disaster recovery and backup are analyzed as follows.

In the risk analysis, first of all, the risk of quantitative analysis is carried out, the problems may occur in the system are estimated, the existing security measures to deal with the situation are analyzed in detail. Then the corresponding protection measures for different levels of disaster are designed, so as to cope with the possible disaster. In addition, enterprises can also be purchased by the way of insurance to transfer risk, so as to reduce the loss of business [6].

In the business impact analysis, it is needed to carry on the analysis to the degree of importance of the data in the power supply enterprises system, and then through the comprehensive consideration of the estimated and unpredictable loss, so as to provide decision-making basis for the power supply enterprise system to withstand the disaster losses.

In the enterprise environment analysis of disaster recovery, in order to obtain the gap between the current environment and the restore disaster of the system, it is needed to analyze and compare the existing conditions and business impact of power supply enterprises. Among them, the most important is the analysis of the resources needed in power supply enterprises [7]. Through the analysis of the disaster recovery environment of enterprise to get all data resources of the enterprise.

In the formulation of disaster recovery strategy process, the risk analysis, enterprise investment capacity and enterprise environment analysis are needed to be integrated, then the disaster recovery strategies and measures for different period are carried out [8]. In addition, the structure of power supply enterprises and the human resources are adjusted to adapt to the disaster recovery strategy.

In the design of the disaster recovery plan, it is necessary to consider the disaster recovery time, disaster recovery scheme of implementation and maintenance.

In the design of business continuity process, in order to guarantee the information system disaster recovery scheme can be recovered after the disaster, at the same time, ensure the recovery of other auxiliary facilities in the business system (including office facilities, department agencies, personnel scheduling and so on) [9].

The last step is the management and testing of business continuity and disaster recovery process. The purpose of the test of the disaster recovery scheme is for finding the problems in the disaster recovery plan from the test results, then according to the problems to carry out the corresponding improvement, and make the disaster recovery scheme more perfect [10].

\section{Methodology}

\subsection{Standard of Disaster Recovery Plan}


In order to ensure the accuracy and reliability of disaster backup scheme, this paper firstly analyzes the nature and types of the disaster that may occur in the information system of power supply enterprises. The recovery rate of the system and the degree of recovery of the data are analyzed. In addition, it is necessary to consider the cost of system implementation issues, current related technologies and constraints.

\subsection{Design of the Backup System}

With the rapid development of power grid enterprise, enterprise database backup has become one of the most important factors that influence the enterprise development. Backup for disaster recovery of power supply enterprise database has a most direct influence on the enterprise information section, even has a crucial role in the development of the whole enterprise. Disaster recovery backup takes over the data backup and application, at the same time orders the enterprise organization, and designs the recovery process. The purpose of which is to ensure that the safety of its database data when the power supply enterprise information system occurs disaster, and then carry on the data recovery, so as to ensure the normal operation of the system. The organizational structure of the backup system designed in this paper as shown in Fig. 1.

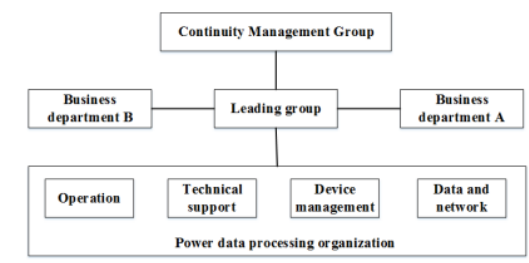

Fig. 1. Organizational structure of disaster backup system

\subsection{Realization of the Disaster Recovery Backup System}

The implementation process of the power supply enterprise data backup system includes anti impact ability of risk analysis, business analysis, business disaster recovery environment analysis and strategy of disaster recovery, disaster recovery scheme of design and management and testing business continuity processes and disaster recovery scheme is formulated.

\section{Result Analysis and Discussion}

In order to verify the effectiveness of the system, this paper takes a power supply enterprise as an example to carry out the simulation test. In the experiment, the database server configurations are the same: P41.80GHz processor, 512MB memory, IDE 40GB hard drive, Windows 7 operating system, 9i Oracle database system. The bandwidth controller software is used to control the bandwidth of the network, and the simulation experiment is carried out under the condition of $3 \mathrm{Mbps}$. In order to simulate the real situation of the database application, the inserting, modifying, deleting and other operations are carried out on the local database $\mathrm{P}$, so as to access to the data generated traffic flow and backup traffic changes curve of $\mathrm{P}$, as shown in Fig. 2. 


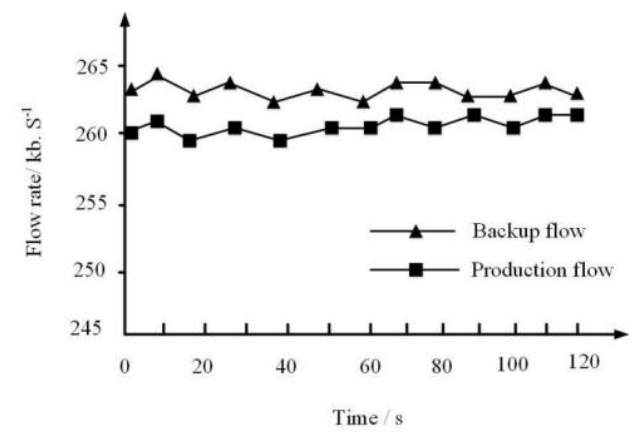

Fig. 2. $P$ Data generated traffic flow and back up traffic change curve of $P$

Backup flow is slightly greater than the generated traffic, which shows that the amount of data changes of $\mathrm{P}$ is copied to $\mathrm{A} 1, \mathrm{~A} 2$ in time, if the $\mathrm{P}$ occurs disaster, $\mathrm{P}$ data will not be lost. The experimental results show that in the slow non steady line, it is a good method to carry on the mufti point and mufti hop database remote disaster recovery and backup, and achieve zero data loss.

\section{Conclusion}

With the rapid development of power grid enterprise, enterprise database backup has become one of the most important factors in the development of the enterprises. In order to ensure the safety of mass data in the information system of the power supply enterprise, the information data backup system for power supply enterprises is designed in this paper according to the selection criteria of the disaster recovery plan, and the risk analysis, strategy formulation, the disaster recovery scheme and other 7 aspects in the disaster recovery and backup scheme are elaborated in detail. The experimental results show that this system can be used for the power supply enterprise data backup, and achieve the zero data loss, so as to ensure the security of information. However, subject to the limitations of my profession, the application of this study in practice is still less, this part should be further in-depth studied.

\section{References}

[1]Xiong wen. Guangzhou power grid dispatching automation system of disaster recovery. Relay, Vol. 32, No. 20, pp. 60-61. (2005)

[2] Chen Lantao, Hu Yonghua. The Yunnan Power Grid Co research and construction of disaster recovery system. Electric power information, Vol. 5, pp. 29-32. (2010)

[3]Wang Muwei, Liu Wenjun, Hu Hongyan. The study of Henan electric power communication network construction of. Power system communication, Vol. 32, No. 11, pp. 13-16. (2011)

[4]Loushu hydrogen, Peng Zhexu. Study on Ji'an dispatch automation system safety and disaster recovery construction. Central China electric power, No. 5, pp. 72-74. (2010) 
[5]Zhang Liang, Xiao Liang Bi, GE Zhaoqiang, etc. Analysis of power dispatching automation system for disaster recovery and backup technology. East China electric power, Vol. 41, No. 6, pp. 1252-1256. (2013)

[6]Abbey C, Cornforth D, Hatziargyriou N, et al. Powering through the storm: microgrids operation for more efficient disaster recovery. Power and Energy Magazine, IEEE, Vol. 12, No. 3, pp. 67-76. (2014)

[7]Sakurai M, Watson R T, Abraham C, et al. Sustaining life during the early stages of disaster relief with a frugal information system: learning from the great east Japan earthquake. Communications Magazine, IEEE, Vol. 52, No. 1, pp. 176-185. (2014)

[8]Tuna G, Nefzi B, Conte G. Unmanned aerial vehicle-aided communications system for disaster recovery. Journal of Network and Computer Applications, Vol. 41, pp. 27-36. (2014)

[9]Huling D, Miles S B. Simulating disaster recovery as discrete event processes using python. Global Humanitarian Technology Conference (GHTC), pp. 248-253. (2015)

[10]Colman-Meixner C, Tornatore M, Mukherjee B. Cloud-Network Disaster Recovery against Cascading Failures. IEEE Global Communications Conference (Globecom), pp. 1-5. (2015) 